\title{
Comparative Assessment of Cutoffs for the Cardio-Ankle Vascular Index and Brachial-Ankle Pulse Wave Velocity in a Nationwide Registry: A Cardiovascular Prognostic Coupling Study
}

\author{
Tomoyuki Kabutoya Kazuomi Kario \\ Division of Cardiovascular Medicine, Department of Medicine, Jichi Medical University School \\ of Medicine, Shimotsuke, Japan
}

\section{Keywords}

Arteriosclerosis · Arterial stiffness · Pulse wave velocity

\begin{abstract}
Pulse wave velocity (PWV) and cardio-ankle vascular index (CAVI) are measurements of arterial stiffness and are widely used as indices of arteriosclerosis. In Japan, brachial-ankle PWV (baPWV) is generally used to measure PWV, and while the reference value for this parameter is specified in Japanese guidelines for the noninvasive vascular function test, the CAVI reference value has not been standardized. We measured CAVI in 4,545 patients with at least one cardiovascular risk factor, and baPWV in 1,737 of these 4,545 patients on the same day as a part of an ongoing nationwide registry. The association between CAVI and baPWV was positive and significant $(r=0.50, p<0.001)$. The CAVI corresponding to baPWV $14 \mathrm{~m} / \mathrm{s}$ derived from the regression line was 8.303 and the CAVI corresponding to baPWV $18 \mathrm{~m} / \mathrm{s}$ was 9.059 . The percentages of patients with low risk (CAVI <8.303), medium risk (CAVI 8.303-9.058) and high risk (CAVI $\geq 9.059$ ) were $35.0,23.0$, and $42.0 \%$ in 4,545 patients who underwent CAVI, respectively. The percentages of patients with baPWV $<14 \mathrm{~m} / \mathrm{s}$, baPWV 14-18 m/s and baPWV $\geq 18 \mathrm{~m} / \mathrm{s}$ were $22.9,47.3$, and $29.8 \%$ in 1,737 patients who underwent both baPWV and CAVI, respectively. The average baPWV in low-risk patients (CAVI <8.303, $n=642$ ) was $14.97 \pm 2.91$ $\mathrm{m} / \mathrm{s}$, that in medium-risk patients (CAVI 8.303-9.058, $n=408$ ) was $16.12 \pm 2.80 \mathrm{~m} / \mathrm{s}$, and that in high-risk patients (CAVI $\geq 9.059, n=687$ ) was $18.40 \pm 3.51 \mathrm{~m} / \mathrm{s}$. CAVI $<8.303$ corresponded to a baPWV cutoff of $14 \mathrm{~m} / \mathrm{s}$, and CAVI $\geq 9.059$ corresponded to a baPWV cutoff of $18 \mathrm{~m} / \mathrm{s}$. The results of this ongoing prospective study are expected to confirm the association between the CAVI reference value and cardiovascular events.

(C) 2018 S. Karger AG, Basel
\end{abstract}


Kabutoya and Kario: Cutoff Value of CAVI

\section{Introduction}

Pulse wave velocity (PWV) is widely used as an index of arteriosclerosis and as a measure of arterial stiffness [1]. Brachial-ankle PWV (baPWV) is widely used to measure PWV in Japan, and more than 12,000 devices are currently available for this purpose. According to the Japanese guidelines for the noninvasive vascular function test, PWV $>18 \mathrm{~m} / \mathrm{s}$ represents a risk of cardiovascular events based on the results of the Hisayama Study [2]. A baPWV level of $14 \mathrm{~m} / \mathrm{s}$ corresponds to moderate risk of cardiovascular events based on the Framingham risk score [3], as well as an increased risk of hypertension onset [4]. Therefore, lifestyle amelioration is recommended in patients with baPWV $\geq 14 \mathrm{~m} / \mathrm{s}$.

The cardio-ankle vascular index (CAVI) is consistent with stiffness parameter $\beta$ and is an index that evaluates artery stiffness theoretically not affected by blood pressure [5]. No reference value is currently available for CAVI in the Japanese guidelines, although a reference value has been established for baPWV.

The aim of this study was to compare CAVI and baPWV measured in the same individuals in a large-scale clinical study, and to assess the CAVI cutoff values corresponding to baPWV 14 and $18 \mathrm{~m} / \mathrm{s}$.

\section{Methods}

Subjects

This study was performed as part of the Coupling Registry, a cardiovascular prognostic coupling study in Japan. The protocol of the Coupling Registry has been registered at the University Hospital Medical Information Network Clinical Trials Registry website under the trial number UMIN000018474. Briefly, the Coupling Registry is a prospective observational evaluation of CAVI values predictive for cardiovascular events in Japanese outpatients with any of the following cardiovascular risk factors at the clinic or hospital: diabetes, glucose tolerance disorder, dyslipidemia, high-normal normotension or grade I-III hypertension ( $>130 / 85 \mathrm{~mm} \mathrm{Hg}$ ), current smoking, renal disease (eGFR $\leq 60$ or positive proteinuria), past history of cardiovascular disease (coronary artery disease, cerebrovascular disorder or non-cardiogenic cerebrovascular disorder, aortic dissection, peripheral artery disease, history of hospitalization by heart failure), atrial fibrillation, metabolic syndrome, chronic obstructive pulmonary disease, and sleep apnea syndrome.

The Coupling Registry is a nationwide multicenter prospective cohort study with a follow-up period of 7 years. All subjects underwent CAVI, and baPWV measurement was conducted as a substudy.

The ethics committee of the internal review board of the Jichi Medical University School of Medicine approved the protocol. The study protocol was registered on the clinical trials registration site, the University Hospital Medical Information Network Clinical Trials Registry (UMIN-CTR), under registration number UMIN000018474. Written informed consent was obtained from all patients enrolled in this study.

\section{Measurement of CAVI and baPWV}

We measured CAVI in 4,545 patients. CAVI was measured automatically using a VaSera VS-1000 device (Fukuda Denshi, Tokyo) with the patient in the recumbent position. The CAVI value was calculated as the average of the right and left CAVI values. We defined patients with CAVI $<8.303$ as low-risk patients, and patients with CAVI $\geq 9.059$ as high-risk patients according to the regression line between baPWV and CAVI.

The baPWV was measured in 1,737 patients of these 4,545 subjects on the same day and under the same conditions as CAVI measurement. baPWV was measured using the volume plethysmographic method with previously validated equipment (form PWV/ABI; Omron Healthcare Co., Ltd.). We used the mean of the right and left baPWV values for the analysis.

Statistical Analysis

Data are shown as the mean \pm SD or as a percentage. The association between CAVI and baPWV was assessed using Spearman's correlation coefficient. Computer software (SPSS version 20.0; SPSS Inc., Chicago, IL, USA) was used for the analyses. A probability value $<0.05$ was considered statistically significant. 


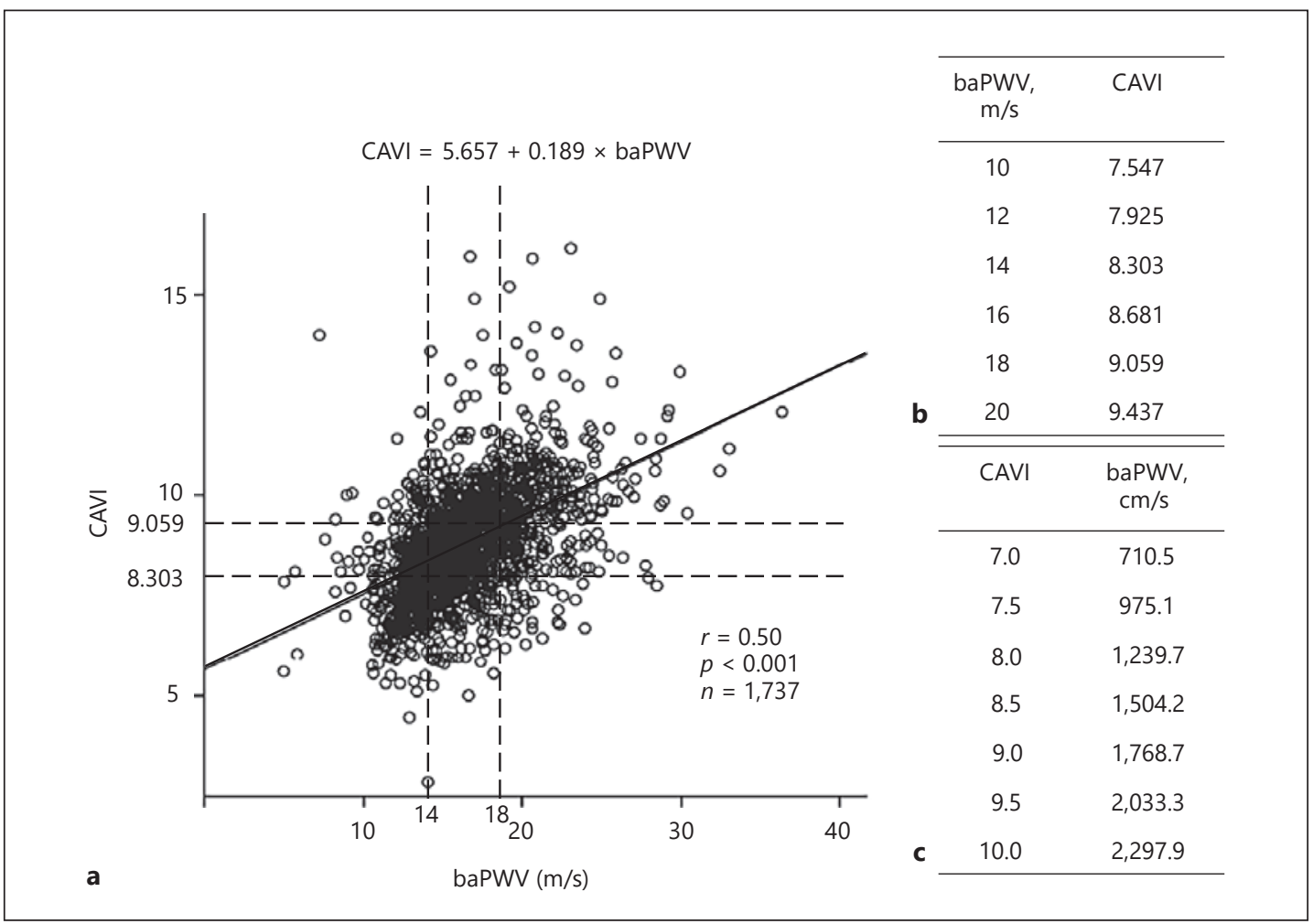

Fig. 1. a The association between cardio-ankle vascular index (CAVI) and brachial-ankle pulse wave velocity (baPWV). b Conversion table from baPWV to CAVI. c Conversion table from CAVI to baPWV.

Table 1. Patient characteristics $(n=4,545)$

\begin{tabular}{lc} 
Age, years & $69.2 \pm 11.2$ \\
Male, \% & 53 \\
Diabetes, \% & 35 \\
Hyperlipidemia, \% & 58 \\
Hypertension, \% & 83 \\
Smoking, \% & 10 \\
Median CAVI (interquartile, 25-75\%) & $8.85(7.98-9.65)$ \\
Median baPWV (interquartile, 25-75\%) & \\
$\quad(n=1,737)$ & $1,615(1,417-1,857)$ \\
\hline
\end{tabular}

CAVI, cardio-ankle vascular index; baPWV, brachial-ankle pulse wave velocity.

\section{Results}

Patient characteristics are shown in Table 1. Average age was $69.2 \pm 11.2$ years, and percentage of males was 53\%. The median of CAVI was 8.85 and that of baPWV was 16.15 $\mathrm{m} / \mathrm{s}$.

\section{Association between CAVI and baPWV}

The association between CAVI and baPWV is shown in Figure 1a. The association was positive and significant $(n=1,737, r=0.50, p<0.001)$, but there were many cases with divergence between CAVI and baPWV. 


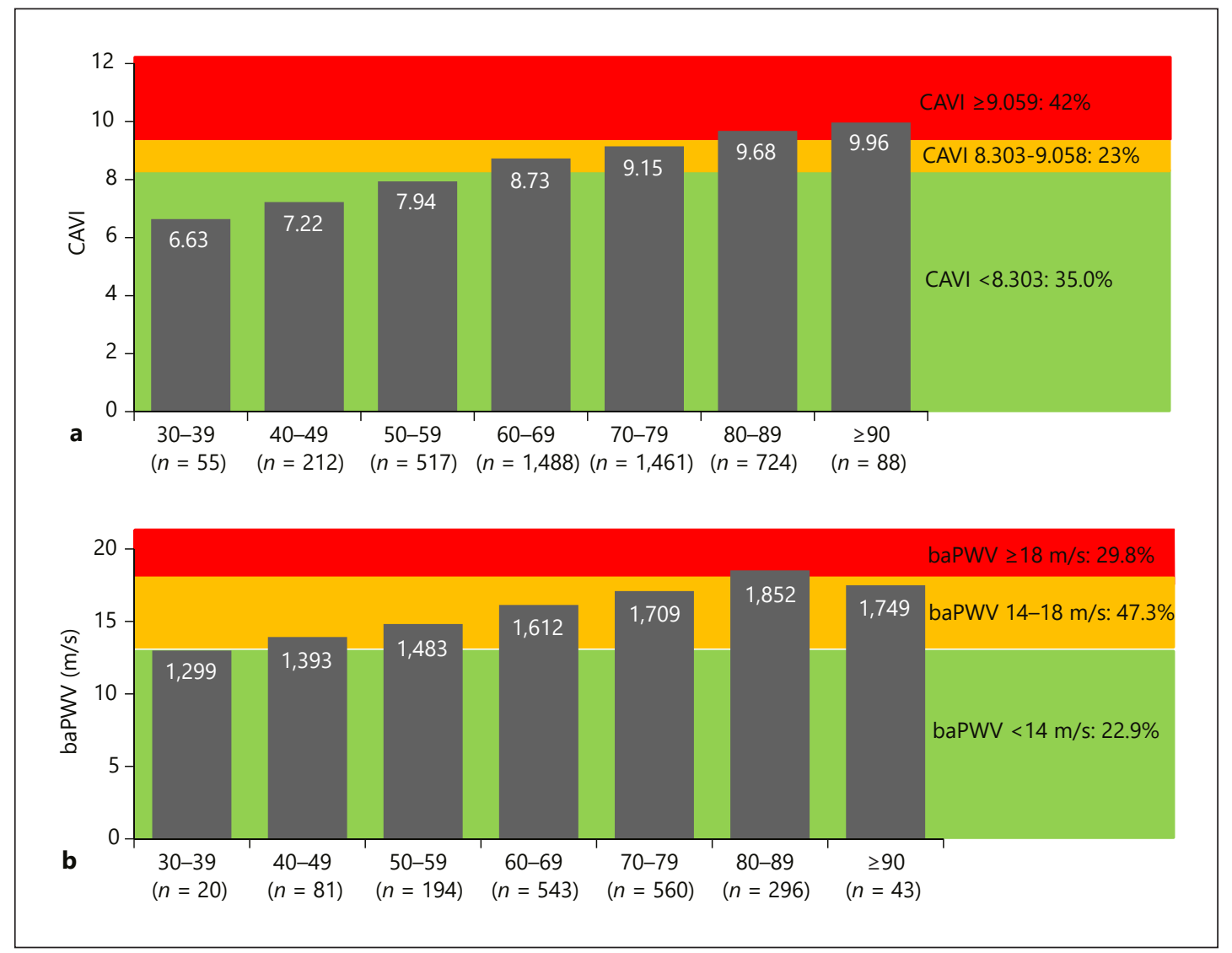

Fig. 2. a The distribution of cardio-ankle vascular index (CAVI). b The distribution of brachial-ankle pulse wave velocity (baPWV).

The CAVI corresponding to baPWV $14 \mathrm{~m} / \mathrm{s}$ derived from the regression line was 8.303 and the CAVI corresponding to baPWV $18 \mathrm{~m} / \mathrm{s}$ was 9.059. The conversion tables from baPWV to CAVI and from baPWV to CAVI are shown in Figure $1 \mathrm{~b}$ and $\mathrm{c}$.

\section{Assessment of the Cutoff Level in CAVI}

The percentages of patients with low risk (CAVI <8.303), medium risk (CAVI 8.3039.058), and high risk (CAVI $\geq 9.059$ ) were $35.0,23.0$, and $42.0 \%$ in 4,545 patients who underwent CAVI, respectively. The association between age and CAVI is shown in Figure 2a $(n=4,545)$. Aging is linearly associated with an increase in CAVI. The average of CAVI in the group aged 60-69 was higher than 8.303, and the average of CAVI in the group aged 80-89 was $\geq 9.059$, which was classified as a high risk for cardiovascular events (Fig. 2a).

The percentages of patients with baPWV $<14 \mathrm{~m} / \mathrm{s}$, baPWV $14-18 \mathrm{~m} / \mathrm{s}$, and baPWV $\geq 18$ $\mathrm{m} / \mathrm{s}$ were $22.9,47.3$, and $29.8 \%$ in 1,737 patients who underwent both baPWV and CAVI, respectively. Aging was also linearly associated with an increase in baPWV; the average baPWV in the group aged 50-59 was more than $14 \mathrm{~m} / \mathrm{s}$, and that in the group aged 80-89 was more than $18 \mathrm{~m} / \mathrm{s}$ (Fig. $2 \mathrm{~b}$ ).

The average baPWV in low-risk patients (CAVI <8.303, $n=642$ ) was $14.97 \pm 2.91 \mathrm{~m} / \mathrm{s}$, that in medium-risk patients (CAVI 8.303-9.058, $n=408$ ) was $16.12 \pm 2.80 \mathrm{~m} / \mathrm{s}$, and that in high-risk patients (CAVI $\geq 9.059, n=687$ ) was $18.40 \pm 3.51 \mathrm{~m} / \mathrm{s}$. 


\section{Discussion}

The main findings of this study were as follows: (1) the association between CAVI and baPWV was positive and significant, but many of the cases showed divergence between CAVI and baPWV; (2) the CAVI corresponding to baPWV $14 \mathrm{~m} / \mathrm{s}$ derived from the regression line was 8.303 and the CAVI corresponding to baPWV $18 \mathrm{~m} / \mathrm{s}$ was 9.059 .

In this study, CAVI was strongly associated with baPWV. To the best of our knowledge, this is the first large clinical study to investigate the CAVI reference value in comparison with baPWV. Gomez-Sanchez et al. [6] investigated the association between adiposity measures using a CAVI with baPWV in Caucasian adults $(n=2,354)$ with intermediate cardiovascular risk. The average of CAVI was 8.8 and the average of baPWV was 14.9, but subjects in the study were European, and the association between CAVI and baPWV was not investigated. Kusunose et al. [7] investigated CAVI values in 114 patients who had at least 2 cardiovascular risk factors. The mean baPWV value was $16.9 \mathrm{~m} / \mathrm{s}$, the mean CAVI value was 8.5, and PWV and CAVI were correlated $(r=0.69, p<0.001)$. However, the study had only a small number of subjects with very high risk, and the authors did not indicate the CAVI reference value. Therefore, CAVI must have been affected by other associated factors in our patients. Indeed, since many of our cases exhibited divergence between CAVI and baPWV, studies of the complementary roles of CAVI and baPWV will be needed.

The CAVI corresponding to baPWV $14 \mathrm{~m} / \mathrm{s}$ derived from the regression line was 8.303 . Thus, a CAVI value of 8.303 is considered to be an appropriate cutoff value separating low and medium risk. The CAVI corresponding to baPWV $18 \mathrm{~m} / \mathrm{s}$ was 9.059. Saji et al. [8] compared arteriosclerotic indicators in patients with ischemic stroke. The cutoff values of baPWV and CAVI for detection of arteriosclerotic ischemic stroke were $18.3 \mathrm{~m} / \mathrm{s}$ and 9.5. These levels seemed to be too high for the cutoff level of CAVI. Chung et al. [9] showed that patients with CAVI $>9.0$ had a greater incidence of cardiovascular events than those with CAVI $\leq 9.0$ (HR $1.18, p=0.049$ ) in diabetes patients, and Sato et al. [10] showed that higher CAVI (CAVI $\geq 10.09$ ) had greater cardiovascular events in metabolic syndrome patients. Kubota et al. [11] showed that high CAVI (CAVI $\geq 10$ ) posed a higher risk of cardiovascular disease events than low CAVI (CAVI <9) did, and thus further discussion about appropriate cutoff values for discriminating low and medium risk will be needed. The average baPWV in patients with CAVI $\geq 9.059$ was more than $18 \mathrm{~m} / \mathrm{s}$, and thus we considered that CAVI 9.059 is acceptable for use in the screening of high-risk patients.

This study was a cross-sectional study, and the subjects were at high cardiovascular risk. To determine appropriate CAVI values for patient groups with different characteristics, it will be necessary to perform prospective studies of healthy subjects.

\section{Conclusion}

CAVI $<8.303$ corresponded to a baPWV cutoff of $14 \mathrm{~m} / \mathrm{s}$, and CAVI $\geq 9.059$ corresponded to a baPWV cutoff of $18 \mathrm{~m} / \mathrm{s}$. The results of our ongoing prospective study are expected to confirm the association between the CAVI reference value and cardiovascular events.

\section{Disclosure Statement}

The authors state that there are no conflicts of interest to report. 


\section{References}

1 Ohkuma T, Ninomiya T, Tomiyama H, Kario K, Hoshide S, Kita Y, Inoguchi T, Maeda Y, Kohara K, Tabara Y, Nakamura M, Ohkubo T, Watada H, Munakata M, Ohishi M, Ito N, Nakamura M, Shoji T, Vlachopoulos C, Yamashina A; Collaborative Group for J-BAVEL (Japan Brachial-Ankle Pulse Wave Velocity Individual Participant Data Meta-Analysis of Prospective Studies): Brachial-ankle pulse wave velocity and the risk prediction of cardiovascular disease: an individual participant data meta-analysis. Hypertension 2017;69:1045-1052.

2 Ninomiya T, Kojima I, Doi Y, Fukuhara M, Hirakawa Y, Hata J, Kitazono T, Kiyohara Y: Brachial-ankle pulse wave velocity predicts the development of cardiovascular disease in a general Japanese population: the Hisayama Study. J Hypertens 2013;31:477-483.

3 Yamashina A, Tomiyama H, Arai T, Hirose K, Koji Y, Hirayama Y, Yamamoto Y, Hori S: Brachial-ankle pulse wave velocity as a marker of atherosclerotic vascular damage and cardiovascular risk. Hypertens Res 2003; 26:615-622.

4 Tomiyama H, Matsumoto C, Yamada J, Yoshida M, Odaira M, Shiina K, Nagata M, Yamashina A: Predictors of progression from prehypertension to hypertension. Am J Hypertens 2009;22:630-636.

5 Hayashi K, Yamamoto T, Takahara A, Shirai K: Clinical assessment of arterial stiffness with cardio-ankle vascular index: theory and applications. J Hypertens 2015;33:1742-1757.

6 Gomez-Sanchez L, Garcia-Ortiz L, Patino-Alonso MC, Recio-Rodriguez JI, Rigo F, Martí R, Agudo-Conde C, Rodriguez-Sanchez E, Maderuelo-Fernandez JA, Ramos R, Gomez-Marcos MA; MARK Group: Adiposity measures and arterial stiffness in primary care: the MARK prospective observational study. BMJ Open 2017; 7:e016422.

7 Kusunose K, Sato M, Yamada H, Saijo Y, Bando M, Hirata Y, Nishio S, Hayashi S, Sata M: Prognostic implications of non-invasive vascular function tests in high-risk atherosclerosis patients. Circ J 2016;80:1034-1040.

8 Saji N, Kimura K, Yagita Y, Kawarai T, Shimizu H, Kita Y: Comparison of arteriosclerotic indicators in patients with ischemic stroke: ankle-brachial index, brachial-ankle pulse wave velocity and cardio-ankle vascular index. Hypertens Res 2015;38:323-328.

9 Chung SL, Yang CC, Chen CC, Hsu YC, Lei MH: Coronary artery calcium score compared with cardio-ankle vascular index in the prediction of cardiovascular events in asymptomatic patients with type 2 diabetes. J Atheroscler Thromb 2015;22:1255-1265.

10 Sato Y, Nagayama D, Saiki A, Watanabe R, Watanabe Y, Imamura H, Yamaguchi T, Ban N, Kawana H, Nagumo A, Ohira M, Endo K, Kurosu T, Tomaru T, Shirai K, Tatsuno I: Cardio-ankle vascular index is independently associated with future cardiovascular events in outpatients with metabolic disorders. J Atheroscler Thromb 2016;23:596-605.

11 Kubota Y, Maebuchi D, Takei M, Inui Y, Sudo Y, Ikegami Y, Fuse J, Sakamoto M, Momiyama Y: Cardio-Ankle Vascular Index is a predictor of cardiovascular events. Artery Res 2011;5:91-96. 